\title{
Sustentabilidade urbana no estado do Pará
}

\author{
Urban sustainability in the state of Pará
}

Sostenibilidad urbana en el estado de Pará

Peri Guilherme Monteiro da Silva ORCID: https://orcid.org/0000-0001-7179-2082

Universidade da Amazônia, Brasil

E-mail: peri-guilherme@ hotmail.com

Leonardo Augusto Lobato Bello ORCID: https://orcid.org/0000-0002-2017-5292

Universidade da Amazônia, Brasil E-mail: leonardo.bello@unama.br

Maria Lúcia Bahia Lopes ORCID: https://orcid.org/0000-0002-3718-0434 Universidade da Amazônia, Brasil E-mail: marialucia.bahia@unama.br

Rosália do Socorro Silva Corrêa ORCID: https://orcid.org/0000-0003-0438-8148 Universidade da Amazônia, Brasil E-mail: rosalia@hotmail.com

\begin{abstract}
Resumo
$\mathrm{O}$ crescimento desordenado cria disparidades sociais, além de degradação em sistemas naturais e artificiais. Esses problemas se atenuam com o desenvolvimento urbano, impedindo de uma cidade ser sustentável, diminuindo a qualidade de vida, não irá existir equidade social, assim como o aumento da poluição e dos índices de violência, para alcançar sustentabilidade um município deve estar munido dados e informações que detectem pontos a serem melhorados no desenvolvimento, tendo em vista essa situação este artigo objetivou-se em realizar um ranking de sustentabilidade com índices que representam a realidade urbana de municípios paraenses, e analisar individualmente cada local. Os municípios paraenses analisados precisam traçar estratégias para melhoria da sustentabilidade urbana, pois não apresentaram bons resultados quando analisados individualmente nos parâmetros social, mobilidade e acessibilidade, econômico e social.
\end{abstract}

Palavras-chave: Sustentabilidade; Desenvolvimento urbano; Índices urbanos.

\section{Abstract}

Disorganized growth creates social disparities, in addition to degradation in natural and artificial systems. These problems are attenuated with urban development, preventing a city from being sustainable, reducing the quality of life, there will be no social equity, as well as increased pollution and violence rates, to achieve sustainability a municipality must be equipped with data and information that detects points to be improved in development, in view of this situation, this article aimed to carry out a sustainability ranking with indexes that represent the urban reality of Pará municipalities, and to analyze each location individually. The analyzed Pará municipalities need to outline strategies for improving urban sustainability, as they did not present good results when analyzed individually in the social, mobility and accessibility, economic and social parameters.

Keywords: Sustainability; Urban development; Urban índices.

\section{Resumen}

El crecimiento desordenado crea disparidades sociales y degradación de los sistemas naturales y artificiales. Estos problemas se atenúan con el desarrollo urbano, impidiendo que una ciudad sea sostenible, disminuyendo la calidad de vida, no habrá equidad social, así como el aumento de las tasas de contaminación y violencia, para lograr la sostenibilidad un municipio debe contar con datos e información que Detectar puntos a mejorar en el desarrollo, ante esta situación, este artículo tuvo como objetivo realizar un ranking de sostenibilidad con índices que representen la realidad urbana de los municipios de Pará, y analizar individualmente cada localidad. Los municipios analizados en Pará necesitan diseñar estrategias para mejorar la sostenibilidad urbana, ya que no presentaron buenos resultados cuando se analizaron individualmente en términos de parámetros sociales, de movilidad y accesibilidad, económicos y sociales.

Palabras clave: Sostenibilidad; Desarrollo urbano; Índices urbanos. 


\section{Introdução}

As cidades passaram por um desenvolvimento não planejado que resultou em modificações nos ambientes urbanos, em uma perspectiva ambiental, existe o aumento da degradação das áreas urbanas e o esgotamento de sistemas naturais, assim como depredação dos artificiais.

Esse processo de urbanização acelerado e sem nenhum planejamento tem como consequência não somente problemas estruturais ao meio, mas também conflitos sociais, aumento da poluição e da violência, dentre outros, resultando em espaços urbanos com o desenvolvimento comprometido (Amadeo et al., 2017; Salles et al., 2019).

Passos, Silveira, Pita, Braga e Silveira (2013) destaca que a expansão urbana quando ocorre de forma desequilibrada os processos podem resultar em condições que favoreçam ao aumento de desigualdades sociais e espaciais, então há a necessidade de uma profunda reflexão sobre o crescimento urbano e sua gestão.

Percebe-se que a gestão deve estar munida de dados e informações que detectem pontos a serem melhorados no desenvolvimento de uma cidade, ação necessária para tornar-se um centro urbano sustentável, para tal situação existem índices e taxas de diferentes dimensões, mas é necessário criar um conjunto que as integre e aponte onde deve ser realizada uma ação.

A política urbana tem de responsabilizar-se do direito sustentável (Brasil, 2001), objetivando-se em ordenar o desenvolvimento urbano, entendendo a cidade como um objeto de função social, deve ser dado o direito à terra urbana, saneamento ambiental, infraestrutura, transporte, trabalho e lazer.

A importância de pensar na sustentabilidade urbana são os resultados de qualidade de vida, promoção de equidade social, assim como na diminuição da poluição e índices de violência e homicídios, em que a gestão pode criar recursos eficazes gerando crescimento a toda população envolvida, ou seja, garantir aos cidadãos condições mínimas de existência para uma vida digna (Ferreira et al., 2020). Segundo Zeng et al, (2016) há a interação entre urbanização e sustentabilidade e é necessário compreendê-la, pois envolve o processo social e o uso da terra.

A gestão urbana necessita de indicadores de sustentabilidade que retratem condições ambientais, econômicas, culturais e institucionais, mas não podem ser apenas um conjunto de dados dispostos sem ordem, pois é necessário que possam orientar e sejam passíveis de análise (Alves et al., 2016), com o intuito de analisar a sustentabilidade e promovê-la ferramentas de avaliação são criadas e aperfeiçoadas, assim como modelos (Martins \& Cândido, 2015). Diante deste contexto questiona-se como estão os cinco municípios paraenses com o maior número de habitantes em relação a sustentabilidade urbana?

Tendo em vista a necessidade de promoção de desenvolvimento sustentável este artigo objetiva-se em analisar municípios paraenses a partir de índices de sustentabilidade urbana e criar um ranking, para realizar comparação entre eles e saber o local que apresenta a melhor situação e possibilitar análises individuais de cada cidade em quatro dimensões: social, mobilidade e acessibilidade, ambiental e econômica.

\section{Metodologia}

\subsection{Localização Geográfica}

Para este estudo foram considerados os cinco municípios com maior número de habitantes no ano de 2020 no estado do Pará, conforme a previsão populacional do IBGE 2019, a localização de cada um dos municípios é exposta pela Figura 1. 


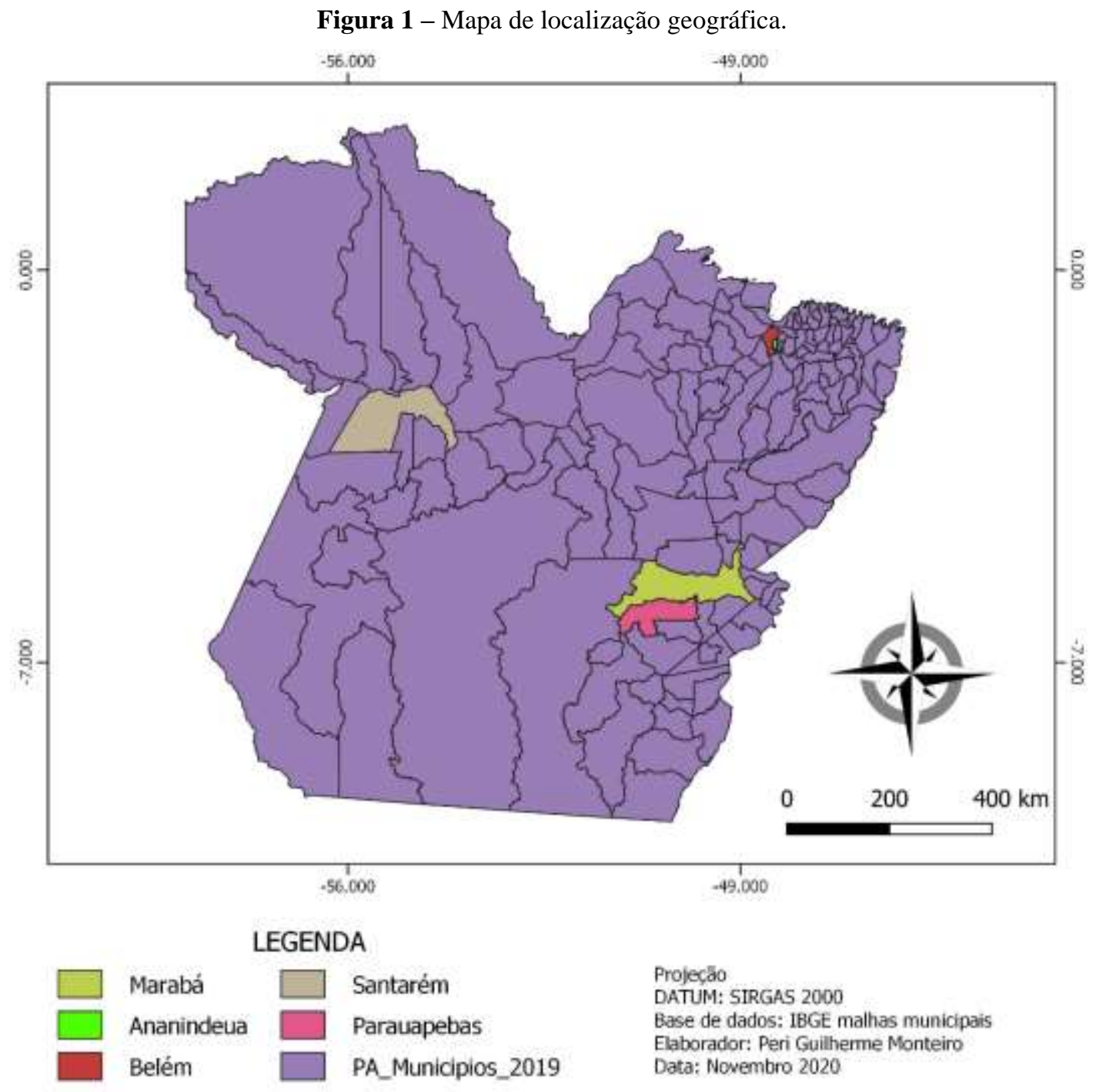

Fonte: Autores (2020).

Conforme o mapa (Figura 1) verifica-se que os cinco municípios estão em regiões distantes, mas quatro dos cinco são limítrofes, sendo Marabá e Parauapebas, Belém e Ananindeua, esses dois últimos estão em processo de conurbação, pois fazem parte da Região Metropolitana de Belém.

\subsection{Processo Metodológico}

Os índices adotados foram baseados nos estudos de Amadeo et al. (2017), Pereira e Vieira (2016) e Ferreira e Vieira (2018). O sistema de ranqueamento foi fundamentado na pesquisa de Amadeo et al. (2017) que consistia em pesos dados a cada indicador, porém optou-se por escolher uma valoração variando no intervalo contido de +1 a -1 , utilizando as seguintes grandezas: $+1,+0,5,0,-0,5,-1$, em que recebia o maior valor o município com a melhor condição e valor inferior àquele em condições de precariedade.

O processo de avaliação de sustentabilidade dos 5 municípios utilizados neste estudo foi baseado no ranqueamento de cada índice, isto é, em cada tópico analisado foi dada uma pontuação aqueles que obtinham a melhor situação, esta estratégia é ilustrada pela Tabela 1, em que são demonstrados valores e posições, complementando com a valoração dada a cada local. 
Tabela 1 - Representação do ranqueamento de sustentabilidade.

\begin{tabular}{|c|c|c|c|c|c|}
\hline Município & \begin{tabular}{lr}
\multicolumn{2}{l}{ População } \\
atendida & por coleta \\
de & Resíduos \\
Sólidos & Urbanos \\
(RSU) & \\
\end{tabular} & Valores & Município & $\begin{array}{l}\text { População atendida com } \\
\text { abastecimento de água }\end{array}$ & Valores \\
\hline Belém & 0,9399999 & 1 & Parauapebas & 0,929338272 & 1 \\
\hline Ananindeua & 0,9975397 & 0,5 & Belém & 0,722263254 & 0,5 \\
\hline Santarém & 0,7325344 & 0 & Santarém & 0,527215424 & 0 \\
\hline Marabá & 0,9088067 & $-0,5$ & Marabá & 0,352516746 & $-0,5$ \\
\hline Parauapebas & 0,9499955 & -1 & Ananindeua & 0,335662857 & -1 \\
\hline Município & Total & & & Ranking final & \\
\hline Belém & 1,5 & & & Belém & $1^{\mathrm{o}}$ \\
\hline Ananindeua & $-0,5$ & & & Santarém & $2^{o}$ \\
\hline Santarém & 0 & & & Parauapebas & $3^{\circ}$ \\
\hline Marabá & -1 & & & Ananindeua & $4^{\circ}$ \\
\hline Parauapebas & 0 & & & Marabá & $5^{\circ}$ \\
\hline
\end{tabular}

Fonte: Autores (2020).

Conforme o processo metodológico deste artigo representado na Tabela 1 em que há uma representação do cálculo a ser realizado (somatório dos pontos recebidos nas variáveis analisadas) tem-se Belém como a melhor situação de sustentabilidade ambiental, pois a soma das duas variáveis utilizadas na exemplificação dá a este município o total de 1,5, colocando-o como primeiro na situação de exemplo da Tabela 1.

Com este processo metodológico é possível visualizar os pontos que necessitam passar por melhorias nos municípios estudados e como se encontram em relação a sustentabilidade urbana quando em comparações municipais. É importante destacar que mesmo terminando com uma boa pontuação e possivelmente ficando em uma boa posição no ranking não representa a negação de necessidade de melhoria ou de planejamento municipal quanto a sustentabilidade.

\subsection{Base de dados}

As fontes para criação do banco de dados do artigo são apresentadas no Quadro 1, foram retirados do Instituto Brasileiro de Geografia e Estatística (IBGE), do Anuário Estatístico do Pará de responsabilidade da Fundação Amazônia de Amparo a Estudos e Pesquisas (FAPESPA), Agência Nacional de Águas (ANA) e Sistema Nacional de Informações sobre Saneamento (SNIS). A partir dos dados disponíveis foram elaborados agrupamentos, chamados de dimensões, sendo: Mobilidade e Acessibilidade, Econômica, Social e Ambiental. 
Quadro 1 - Fonte dos indicadores.

\begin{tabular}{|c|c|c|}
\hline Dimensão & Indicadores & Fonte \\
\hline \multirow{6}{*}{ 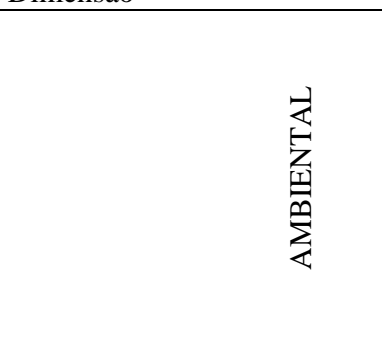 } & Forma de disposição final de RSU ${ }^{1}$ & Câmaras municipais ${ }^{2}$ \\
\hline & $\begin{array}{l}\text { Taxa de população atendida por coleta de } \\
\text { RSU }^{3}\end{array}$ & SNIS (2018); FAPESPA (2019) \\
\hline & $\begin{array}{lccc}\begin{array}{l}\text { Taxa de população } \\
\text { abastecimento de água }\end{array} & \text { atendida } & \text { com } \\
\end{array}$ & SNIS (2016); FAPESPA (2019) \\
\hline & Taxa de esgoto coletado ${ }^{5}$ & ANA (2013) \\
\hline & $\begin{array}{lccc}\text { Taxa de população } & \text { atendida } & \text { com } \\
\text { esgotamento sanitário }^{6} & & \\
\end{array}$ & SNIS (2016); FAPESPA (2019) \\
\hline & Arborização de vias públicas ${ }^{7}$ & IBGE (2010) \\
\hline \multirow{5}{*}{ 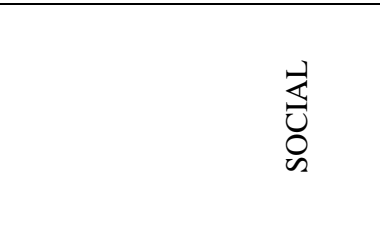 } & Famílias inscritas em programas sociais ${ }^{8}$ & FAPESPA (2019) \\
\hline & Taxa de homicídios por 100.000 habitantes $^{9}$ & FAPESPA (2019) \\
\hline & $\mathrm{N}^{\circ}$ de médicos por 10.000 habitantes ${ }^{10}$ & FAPESPA (2019) \\
\hline & $\begin{array}{l}\text { Taxa de estabelecimentos do ensino } \\
\text { fundamental }^{11}\end{array}$ & FAPESPA (2019) \\
\hline & Taxa de abandono no ensino fundamental ${ }^{12}$ & FAPESPA (2019) \\
\hline \multirow{3}{*}{ 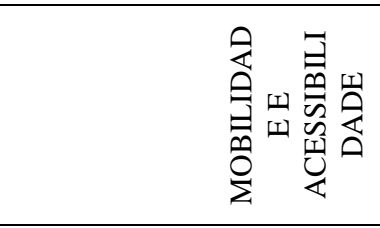 } & $\begin{array}{l}\text { Lei sobre acessibilidade para pessoas } \\
\text { portadoras de deficiências ou com } \\
\text { mobilidade reduzida }^{13}\end{array}$ & Câmaras municipais ${ }^{2}$ \\
\hline & $\begin{array}{lll}\text { O número } & \text { de } & \text { veículos } \\
\text { per capita }^{14} & & \\
\end{array}$ & $\begin{array}{llll}\text { IBGE } & (2020) ; & \text { MINISTÉRIO } & \text { DE } \\
\text { INFRAESTRUTURA } & (2020) & \\
\end{array}$ \\
\hline & Taxa de homicídios no trânsito ${ }^{15}$ & FAPESPA (2019) \\
\hline \multirow{4}{*}{ 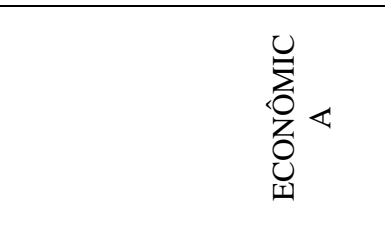 } & Taxa de ocupação & FAPESPA (2019) \\
\hline & População ocupada & IBGE (2018) \\
\hline & 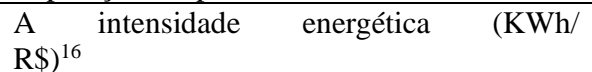 & IBGE (2017) \\
\hline & $\begin{array}{lccc}\text { A } & \text { intensidade no } & \text { uso energético } \\
\text { domiciliar }(\mathrm{KWh} / \mathrm{hab} .)^{17} & & \\
\end{array}$ & FAPESPA (2019); IBGE (2017) \\
\hline
\end{tabular}

Fonte: Autores (2020).

\section{Análise dos Resultados}

A Tabela 2 apresenta os resultados do ranqueamento, tendo em primeiro lugar Belém, a capital paraense, seguida por Ananindeua, dois municípios pertencentes a Região Metropolitana de Belém, na direção contrária com os valores negativos são os municípios do interior paraense, localizados na região sudeste do estado, Marabá e Parauapebas, e o ponto do meio é o município de Santarém, pertencente a outro extremo paraense, a Região Metropolitana de Santarém, ficando no oeste do estado do Pará.

\footnotetext{
${ }^{1}$ Considerou-se a análise em aterros sanitários, aterros controlados e lixões, quando o município apresentar aterro sanitário recebe a pontuação no valor 1 , aterro controlado 0,5 e lixão -1 .

${ }^{2}$ Foram pesquisados os documentos das câmaras municipais para apurar sobre o tema.

${ }^{3}$ Calculou-se a relação percentual de população total prevista no ano de 2018 (FAPESPA) pela população atendida por coleta de RSU no ano de 2018 (SNIS).

${ }^{4}$ Calculou-se a relação percentual de população total prevista no ano de 2016 (FAPESPA) pela população atendida por coleta de RSU no ano de 2016 (SNIS).

${ }^{5}$ Informação fornecida pela Agência Nacional de Águas no Atlas de Saneamento.

${ }^{6}$ Calculou-se a relação percentual de população total prevista no ano de 2016 (FAPESPA) pela população atendida com esgotamento sanitário no ano de 2016 (SNIS).

${ }^{7}$ Informação fornecida pelo Instituto Brasileiro de Geografia e Estatística (IBGE).

${ }^{8}$ Informação fornecida pela FAPESPA para o ano de 2018 denominada: Total de famílias inscritas no Cadastro Único para programas sociais (CadÚnico).

${ }^{9}$ Informação do ano de 2017 fornecida pela FAPESPA.

${ }^{10}$ Informação do ano de 2018 fornecida pela FAPESPA.

${ }^{11}$ Taxa calculada pela proporção do número de escolas do ensino fundamental pelo número de matrículas no ensino fundamental no ano de 2017.

${ }^{12}$ Informação do ano de 2017 fornecida pela FAPESPA.

${ }^{13}$ Pesquisa por instrumentos legais municipais que pautem sobre a promoção de acessibilidade das pessoas portadoras de deficiência ou com mobilidade reduzida, quando o município tiver lei recebe o valor de 1 e quando não tiver -1 .

${ }^{14}$ Dividiu-se a frota total de veículos pela população, ambos para o ano de estudo.

${ }^{15}$ Informação do ano de 2017 fornecida pela FAPESPA.

${ }^{16}$ Dividiu-se o PIB Municipal de 2017 pelo consumo total de energia do mesmo ano.

${ }^{17}$ Dividiu-se o consumo domiciliar em 2017 pela população total para o mesmo ano.
} 
Tabela 2 - Ranking de sustentabilidade urbana.

\begin{tabular}{ccc}
\hline Município & Ranking de sustentabilidade urbana & Pontuação \\
\hline Belém & 1 & 8,5 \\
Ananindeua & 2 & 2,5 \\
Santarém & 3 & 2,5 \\
Parauapebas & 4 & -1 \\
Marabá & 5 & -5 \\
\hline
\end{tabular}

Fonte: Autores (2020).

Nota-se uma diferença entre a capital paraense e os demais municípios, Belém demonstra ser aquela com os melhores valores, logo, pode-se analisar ela como sendo o município mais sustentável dentre os escolhidos para este estudo.

Como mencionado anteriormente, os resultados podem ser utilizados para analisar quais os pontos que os municípios deveriam melhorar, para facilitar essa análise foi realizada a criação do gráfico de radar, utilizando as quatro dimensões (Mobilidade e Acessibilidade, Econômica, Social e Ambiental), essa informação é exposta na Figura 2.

Figura 2 - Radar de índices.

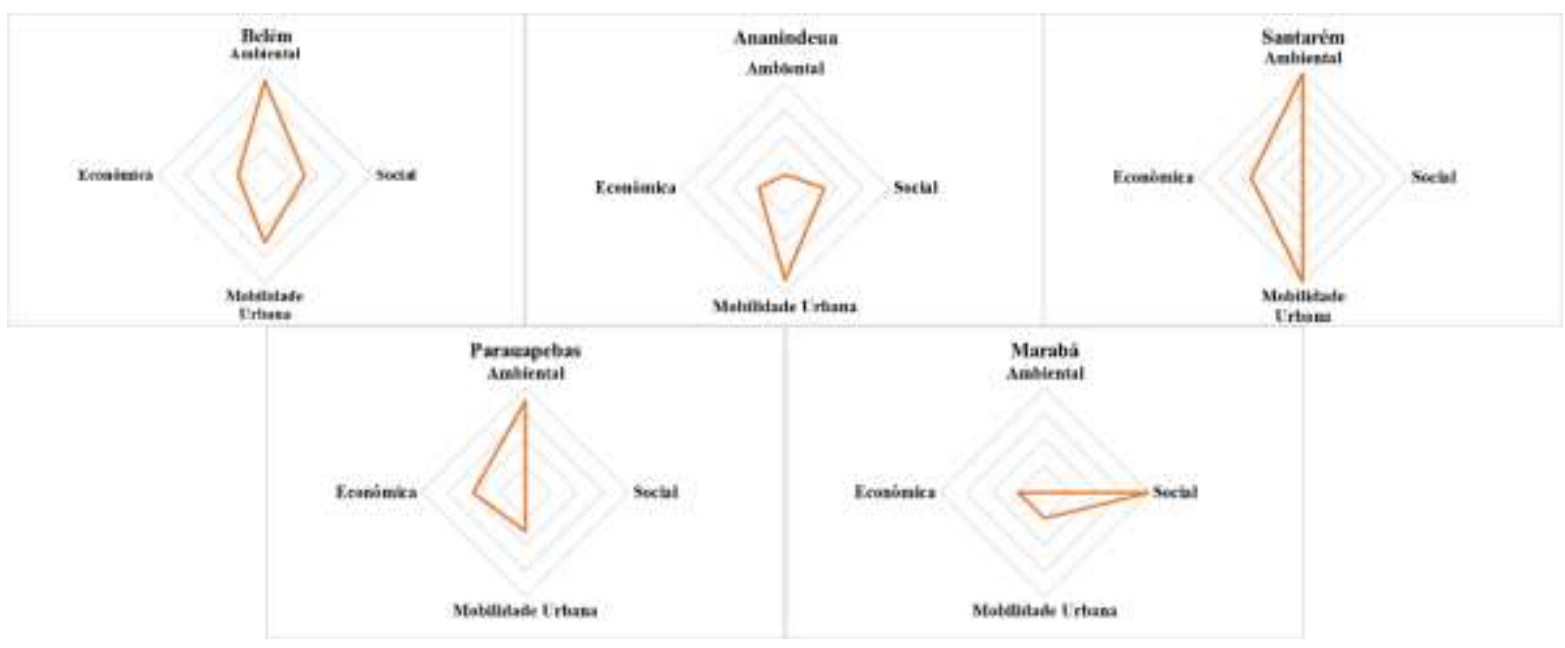

Fonte: Autores (2020).

Os parâmetros de economia e social mostram-se com pouca valoração em Belém, logo, pode-se entender que exista um baixo nível de equidade social e uma pequena taxa de pessoal ocupado, o que pode ser consequente para uma pressão por consumo elétrico (Pereira \& Vieira, 2016) no município. Discutindo-se sobre a situação de população ocupada tem-se uma situação contrária a garantia ao trabalho que é dita pelo estatuto da cidade como uma das diretrizes da política urbana, então deve haver uma programação na agenda municipal para fomentação de atividades geradoras de empregos.

A mobilidade urbana em Belém é um ponto relevante, pois este município apresenta um considerável número de veículos por habitante assim como existem leis de acessibilidade para pessoas com deficiência, porém há uma considerável taxa de homicídios no trânsito, este fator fez com que o índice de mobilidade urbana de Belém sofre-se penalidades, pois a taxa de homicídios é uma situação considerada como de situação indesejável, logo, o município com maior valor nessa variável apresenta a menor nota para ranqueamento.

Ananindeua necessita analisar suas políticas quanto ao setor ambiental, tendo em vista que é um município com uma proporção insular, cerca de 33\% do seu espaço geográfico é composto por ilhas (Ananindeua, 2006), com os dados da Figura 2 tem-se uma situação interessante, pois segundo Santos (2010) o plano diretor de Ananindeua apresenta uma preocupação 
ambiental, porém os índices apresentados expõem que mesmo ocorrendo atenção a esta dimensão no documento, não ocorre o mesmo no mundo real.

O município de Santarém mesmo apresentando pontuação positiva no ranking deve agir sobre a sua situação social, pois foi nessa dimensão que não teve nenhum valor, observando a representação radial não há movimentação nesse parâmetro, logo, torna-se um problema para sustentabilidade, porém Santarém apresenta boas condições ambientais e tem legislações e baixa taxa de violência no trânsito, favorecendo as condições para mobilidade urbana.

A situação de Santarém apresenta uma possível demanda de melhoria e exige uma atenção sobre os fatores econômicos, pois mesmo com uma situação que se pode ser considerada como média (em comparação aos resultados dos demais locais de estudo) há compreensão que deva haver um processo para aumento da taxa de população ocupada, isto é, criação de projetos que fomentem a geração de empregos.

A situação de Parauapebas na sustentabilidade urbana é afetada pela sua condição de mobilidade urbana e social, pois existe uma lei que trata sobre acessibilidade e mobilidade, porém existe uma alta taxa de número de homicídios no trânsito, fator que diminui o potencial de mobilidade urbana.

É interessante analisar a dimensão ambiental em Parauapebas, pois o aterro sanitário existente não atende todos os critérios de operação, sendo na verdade um aterro controlado, motivo que diminui a pontuação desse município. Os demais índices que compõem a dimensão ambiental apresentam valores positivos que melhoram a situação de sustentabilidade municipal.

Em Marabá existe a situação mais crítica no ranqueamento, pois apresenta baixos índices em três das quatro dimensões, a única sobressalente é a social, mas é necessário compreender um município em uma visão holística, cabendo a gestão municipal analisar ações a serem realizadas em todas as dimensões individualmente, mas que possam agregar valores e melhorar as condições defasadas no município.

Marabá é o município que a dimensão social apresenta maiores valores, logo é a que melhor se apresenta nessa situação, demonstrando garantia de direitos básicos ao desenvolvimento urbano segundo os índices apresentados no Quadro 1, mas a condição deste município no sistema de ranqueamento em relação aos demais municípios é abalada em consequência dos demais fatores estarem em condições mínimas, isto é, há necessidade de melhorias nos instrumentos que compõem os itens analisados nos outros índices.

É interessante visualizar que Belém mesmo sendo a capital, nos índices que representam a dimensão social apresenta valores abaixo da cidade com a menor pontuação no ranking de sustentabilidade, Marabá, ou seja, utilizando como base os três índices analisados é perceptível a necessidade de melhoria da gestão pública quanto a parte social em Belém.

Percebe-se que todos os municípios devem passar por aprimoramentos da gestão pública, e uma estratégia que pode e deve ser considerada para alcance de melhorias por cada município nos índices que apresentam baixa valoração é a de realizar análises de beanchmarking para compreender como podem ser utilizadas estratégias dos outros locais de estudo para melhorar sua situação.

\section{Considerações Finais}

Os resultados demonstram que os municípios que apresentam conurbação, Belém e Ananindeua, concentram os melhores índices de sustentabilidade dentre os 5 escolhidos para o estudo, enquanto os localizados na região sudeste do estado do Pará tiveram os piores índices no ranqueamento.

O ranqueamento de sustentabilidade aqui apresentado serve para diagnosticar como está a situação de sustentabilidade urbana de um município, por vezes pode ter um alto valor em um índice o que representa uma boa condição, mas quando em 
comparação aos demais, pode representar a necessidade de que possa crescer, se igualar e ultrapassar o que seria o melhor cenário, em exemplo tem-se a taxa de abastecimento de água, todos os municípios analisados apresentam altas taxas de população atendida, porém, quando comparados existirá o ranqueamento que diagnosticará um município sendo ótimo e os demais em situação de necessidade de melhoria.

É perceptível que nenhum dos municípios analisados apresentam boas condições em todos os parâmetros, principalmente os municípios do interior do estado do Pará em comparação a Belém, capital paraense, e dentre eles a situação menos desejável é a de Marabá, que tem valores baixos em três dos quatro grupos de índices.

Chama-se atenção para necessidade de análise holística dos resultados, pois deve-se considerar um centro urbano como um organismo integrado, isto é, alterações em determinadas condições, podem ser refletidas em outros índices e outras situações municipais.

A proposta de ranqueamento de sustentabilidade urbana foi alcançada, apesar da dificuldade de disposição de dados foi possível criar informações que possam ser utilizadas de forma eficiente pelos gestores municipais para traçar estratégias de melhoria urbana.

\section{Agradecimentos}

O presente trabalho foi realizado com apoio da Coordenação de Aperfeiçoamento de Pessoal de Nível Superior -Brasil (CAPES) - Código de Financiamento 001.

\section{Referências}

Alves, S. F., Barreto A. A., Rodrigues, P. C. H., \& Feliciano, V. M. D. (2016). Indicadores de sustentabilidade para institutos de pesquisa e inovação da área. Brazilian Journal of radiation sciences, 4 (1).

Amadeo, R. M., Soares, P. F., \& Valques, I. J. B. (2017). Proposta de índice de potencial de sustentabilidade urbana: estudo de caso em Pedra Branca-SC. Cidades verdes, 5 (12).

Agência Nacional de Águas. Atlas Esgotos. (2013). http://www.snirh.gov.br/portal/snirh/snirh-1/atlas-esgotos.

Ananindeua. (2006). Lei $n^{\circ}$ 2.237, de 6 de outubro de 2006. Institui o Plano Diretor do Município de Ananindeua e dá outras providências. Ananindeua: Câmara municipal.

Brasil. (2001). Lei n ${ }^{\circ}$ 10.257, de 10 de julho de 2001. Regulamenta os arts. 182 e 183 da Constituição Federal, estabelece diretrizes gerais da política urbana e dá outras providências.

Fundação Amazônia de Amparo a Estudos e Pesquisas. Anuário Estatístico do Pará 2019. <http://www.fapespa.pa.gov.br/menu/163>.

Ferreira, I. T., Panazzolo, M., \& Kohler, V. L. (2020). Cidade sustentável: direito a uma vida urbana digna. Brazilian jornal of development, 6 (8).

Ferreira, A. E. de M., \& Vieira, I. C. G. (2018). Sustentabilidade urbana na região metropolitana de Santarém, Pará, Brasil nos anos 2000 e 2010. Economía, Sociedad y Territorio, 18 (58), p. 763-795.

Instituto Brasileiro de Geografia e Estatística. (2020). Estimativas de População 2020. https://www.ibge.gov.br/estatisticas/sociais/populacao/9103estimativas-de-populacao.html?=\&t=o-que- 0 .

Instituto Brasileiro de Geografia e Estatística. (2018). População ocupada. https://cidades.ibge.gov.br/ >.

Instituto Brasileiro de Geografia e Estatística. (2018). População ocupada. https://cidades.ibge.gov.br/.

Instituto Brasileiro de Geografia e Estatística. (2017). Produto Interno Bruto - PIB. https://www.ibge.gov.br/explica/pib.php.

Ministério de Infraestrutura. (2020). Frota de veículos 2020. https://www.gov.br/infraestrutura/pt-br/assuntos/transito/conteudo-denatran/frota-de-veiculos2020 .

Martins, M. F., \& Cândido, G. A. (2015) Modelo de avaliação do nível de sustentabilidade urbana: proposta para as cidades brasileiras. URBE. Revista Brasileira de Gestão Urbana, 7 (3), p. 397-410.

Passos, L. A.., Silveira, F. A., Pita, A. L. L. R., Braga, C. F. C., \& Silveira, J. A. R. (2012). Processo de expansão versus sustentabilidade urbana: reflexão sobre as alternativas de deslocamento na cidade de João Pessoa, PB. URBE: Revista Brasileira de Gestão Urbana, 4 (1), p. $47-59$. 
Research, Society and Development, v. 11, n. 1, e3711124344, 2022

(CC BY 4.0) | ISSN 2525-3409 | DOI: http://dx.doi.org/10.33448/rsd-v11i1.24344

Pereira, F. da S., Vieira, I. C. G. (2016). Expansão urbana da Região Metropolitana de Belém sob a ótica de um sistema de índices de sustentabilidade. Rev. Ambient. Água, 11 (3).

Salles, M. C. T., Martins, M. de F., \& Cândido, G. A. (2019). Sustentabilidade urbana: uma proposta metodológica para análise e classificação de cidades. Revista ibero-americana de ciências ambientais, 10 (4).

Santos, T. V. dos. (2010). Fronteiras de papel: uma análise da perspectiva metropolitana em planos diretores da região metropolitana de Belém. Dissertação de mestrado em Geografia, Programa de pós-graduação em Geografia, Universidade Federal do Pará, Belém, Brasil.

Sistema Nacional de Informações sobre Saneamento. (2016). Série Histórica 2016. http://app4.mdr.gov.br/serieHistorica/\#.

Sistema Nacional de Informações sobre Saneamento. (2018). Série Histórica 2018. http://app4.mdr.gov.br/serieHistorica/\#.

Zeng, C., Deng X., Dong, J., \& Hu, P. (2016), Urbanization and Sustainability: Comparison of the Processes in “BIC” Countries, Sustainability, 8 (4) pp. 1-18. 